\title{
ORFanID: A Web-Based Search Engine for the Discovery and Identification of Orphan and Taxonomically Restricted Genes
}

4 Richard S. Gunasekera ${ }^{1 *}$, Komal K. B. Raja ${ }^{2}$, Suresh Hewapathirana ${ }^{3}$, Thushara Galbadage ${ }^{4}$,

5 Emanuel Tundrea ${ }^{5}$, Vinodh Gunasekera ${ }^{6}$, and Paul A. Nelson ${ }^{{ }^{*}}$

6

$7 \quad{ }^{1}$ Department of Chemistry, Physics and Engineering, School of Science, Technology \& Health, Biola

8 University, La Mirada, CA

$9 \quad{ }^{2}$ Department of Pathology \& Immunology, Baylor College of Medicine, Houston, TX

$10{ }^{3}$ European Bioinformatics Institute, Wellcome Genome Campus, Hinxton, Cambridgeshire, UK,

$11{ }^{4}$ Department of Kinesiology and Health Science, School of Science, Technology \& Health, Biola

12 University, La Mirada, CA

$13{ }^{5}$ The Ethics and Society Research Centre, Griffiths School of Management and IT, Emanuel University

14 of Oradea, Romania

$15{ }^{6}$ Bioinformatics, Chesalon USA, Inc., Houston TX

16

17 * Correspondence: Corresponding

18 Authors

19 richard.gunasekera@biola.edu

20 paul.alfredp@gmail.com

23 Keywords: ORFanID, orphan gene, ortholog, Taxonomically Restricted Genes (TRG), DNA, 24 protein, bioinformatics 
ORFanID: Web-Based Search Engine

\section{Abstract}

With the multiplicity of genomes sequenced today, it has been shown that significant

30 percentages of genes in any given taxon do not possess orthologous sequences in other taxa. These

31 sequences are typically designated as orphans/ORFans when found as singletons in one species only

32 or taxonomically restricted genes (TRGs) when found at higher taxonomic ranks. Therefore,

33 quantitative and collective studies of these genes are necessary for understanding their biological

34 origins. Currently, orphan gene identifying software is limited, and those previously available are either

35 not functional, are limited in their database search range, or are too complex algorithmically. Thus, an

36 interested researcher studying orphan genes must harvest their data from many disparate sources.

37 ORFanID is a graphical web-based search engine that efficiently finds orphan genes and TRGs at all

38 taxonomic levels, from DNA or amino acid sequences in the entire NCBI database cluster and other

39 large bioinformatics repositories. To our knowledge, this is the first program that allows the

40 identification of orphan genes using both nucleotide and protein sequences in any species of interest.

41 ORFanID identifies genes unique to any taxonomic rank, from species to a domain, using

42 standard NCBI systematic classifiers. The software allows for user control of the NCBI database search

43 parameters. The results of the search are provided in a spreadsheet as well as a graphical display. All

44 the tables in the software are sortable by column, and results can be easily filtered with fuzzy search

45 functionality. In addition, the visual presentation is expandable and collapsible by taxonomy. 


\section{Introduction}

ORFanID: Web-Based Search Engine

Following the introduction of large-scale, high-throughput automated DNA sequencing in the mid1990s, the comparative analysis of whole genomes has revealed that a significant number of

51 protein-coding open reading frames (ORFs) occur only in one species or only in taxonomically

52 restricted groups (TRG's). These TRG's occur at systematic ranks from genus upwards. Species-

53 specific ORFs have been designated as "orphan" (sometimes spelled "ORFan") genes, whereas more

54 widely distributed ORFs, not present universally but only Linnaean taxa, have been designated as

“taxonomically restricted genes" (TRGs). By definition, orphan genes have no counter orthologous

(i.e., homologous) sequences in any other species. Similarly, TRGs are not present in any genomes

outside their respective taxa. For example, a TRG may be found only in the genus Drosophila, but not

in any other Dipteran or in any larger systematic category: Insecta, Arthropoda, and so forth.

Historically, most genetic analyses have omitted the study of orphan and taxonomically

restricted genes and favored focusing on conserved genes, common either to all organisms (e.g., a few

tens of ribosomal sequences) or to broader systematic categories (e.g., the Wnt signaling pathway in

Metazoa), due to the difficulty of studying taxonomically unique ORFs. This is because functional

roles are generally assigned to newly sequenced genes via homology criteria (e.g., existing

annotations). Since conserved genes were straightforward to analyze in evolutionary theory,

systematics, translational and medical research, this focus is easily understood. However, we believe

that understanding orphan genes, which evidence shows (Prabh and Rödelsperger 2016; Wissler et al.

67 2013; Yu and Stoltzfus 2012), are uniquely involved in making one species distinct from another

68 phenotypically, is equally significant.

The last decade has seen a rapidly increasing interest in studying orphan genes (Gao et al. 2020;

Weisman et al. 2020; and Johnson 2018). Genomic sequencing has revealed that significant fractions 


\section{ORFanID: Web-Based Search Engine}

72 species. Therefore, TRGs represent evolutionarily important mediators of phenotypic novelty (Johnson

73 and Tsutsui 2011; Khalturin et al. 2009; Tautz 2011; Tautz and Domazet-Lošo 2011). Previous dogma

74 implied that all genes have descended by modification from the set of coding sequences present in the

75 Last Universal Common Ancestor (LUCA). Thus, the prevailing theory had posited that the duplication

76 of existing genes, followed by a divergence into new functions, brought forth all extant genes (Lupas

77 et al. 2001). This duplication-and-divergence process model predicted that any extant ORF should

78 reveal its history compared to the known universe of other ORFs, given that all ORFs stand on

79 branching lineages descended from their predecessors in LUCA.

However, the finding of orphan genes has led to a changing picture of gene evolution and

81 formation. The novel emergence of genes suggests de novo formation may be a predominant

82 mechanism for gene emergence (Tautz and Domazet-Lošo 2011). This radical shift in thinking has

83 significant ramifications for understanding the nature of genes, the genome's non-coding regions, and

84 fully functional sequences (Tautz 2011). This calls for the re-analysis of existing data and comparative

85 genomic analysis, which can provide new and accurate understandings leading to theories in genetics,

86 genomics, and the tree of life (Ibrahim et al. 2021). In time, the biological understanding of the origins

87 and the functions of orphan genes will have applications in both medicine and evolutionary biology

88 across the tree of life. Therefore, it can be asserted that orphan genes (and TRGs) represent one of the

89 most intriguing aspects of biology, lying at the intersection of genomics, genetics, comparative and

90 structural biology, phylogenetics, and evolution.

91 To study growing numbers of these novel genes across genomes calls for easy-to-use

92 bioinformatics tools that can be utilized by scientists from the life sciences and those with

93 computational backgrounds. Tools have been made available through BLAST (Fischer and Eisenberg

94 1999; Yin and Fischer 2006) and related machine language programs (Ekstrom and Yin 2016). 
ORFanID: Web-Based Search Engine

95 However, web-based, easy-to-use intuitive tools are further necessary and useful for identifying and

96 discovering orphan genes across taxonomic lineage phylostratigraphy - the process of determining the

97 phylogenetic origin of every gene in a genome (Domazet-Lošo et al. 2007).

98 The tool options a researcher can find today to study orphan genes are very limited, simply

99 because most software solutions focus on identifying orthologs or inferencing ortho groups and because

100 their scope is generally limited to proteins. For instance, ORFanFinder (Ekstrom and Yin 2016)

101 functionality is limited to plants, bacteria, and fungi. While the URL provided in the original

102 publication (http://cys.bios.niu.edu/orfanfinder) does not work, it appears that ORFanFinder is active

103 at http://bcb.unl.edu/orfanfinder/ although it does not seem to have been updated. SequenceServer

104 (Priyam et al. 2019) performs blast without classifying the proteins/DNA sequences to taxonomic

105 levels. The software Geneious (Kearse et al. 2012) performs alignment and can build a phylogenetic

106 tree, but identifying orphans using this software may be arduous. Similarly, OrthoFinder (Emms and

107 Kelly 2019) provides the option to use DIAMOND (Buchfink et al. 2015) or it's recommended

108 MMseq2 (Mirdita et al. 2019) is for aligning sequences. OMA orthology (Altenhoff et al. 2018) or the

109 series of analytical resources developed by the Bioinformatics Resource Centers (BRCs) for Infectious

110 Diseases program [bioinformatics tools, workspaces and services for bioinformatics data analysis like

111 AmoebaDB, FungiDB, OrthoMCL (Li et al. 2003)] only show orthologous genes/proteins and do not

112 identify orphan genes. In short, these tools perform alignment or identify conserved genes from the

113 genomes but are not uniquely designed to identify orphans as ORFanID is designed to do.

114 ORFanID's distinctiveness is threefold: (1) Its scope includes processing not just the

115 protein/amino acid sequences but also DNA/nucleotide sequences. (2) Using its built-in homology

116 interpreter and classifier, the search engine provides the taxonomic rank of a gene either as an orphan

117 gene or as a gene restricted to a taxonomic level in the tree of life; (3) As Orphans and TRGs are 
ORFanID: Web-Based Search Engine

118 identified, ORFanID builds its own database with the results of the analysis and provides the researcher

119 with the possibility to mine the data further.

120 2. Methods

\section{$121 \quad 2.1$ Algorithm and Implementation}

122 ORFanID identifies orphan genes and TRGs from a given list of DNA or protein sequences

123 (Figure 1) mainly by accession number. Based on the protein or DNA sequence, detectable homologous

124 sequences are found in the NCBI non-redundant databases using the BLAST alignment tool. All the

125 tools mentioned above recognize NCBI databases are among the most trusted sources. BLAST is a

126 well-established tool, but users acknowledge it is slow in processing as the databases grow rapidly.

127 If the query sequence is a protein, the BLASTP program is used, while the BLASTN program

128 is used for the nucleotide sequences. ORFanID allows the user to submit multiple sequences in FASTA

129 format so that the queries can be queued for processing using software message brokering techniques.

130 The results will be combined into a single blast report in tab-delimited format, downloadable by the

131 user. ORFanID sends a customized BLAST command to retrieve taxonomy IDs of each hit (the

132 "staxid" column of Figure 2A). Using the rank lineage information file provided by the NCBI

133 Taxonomy Database, the ORFanID Homology Interpreter finds the defined taxonomy level (species,

134 genus, family, order, class, phylum, kingdom, and superkingdom) for each sequence found in the

135 BLAST results. (Figure 2B).

136 After finding the complete lineage for the individual homology for each input DNA or protein

137 sequence, the ORFanID Classifier begins searching from the Superkingdom level working towards the

138 Species level to find common ancestors (Figure 3). The taxonomic rank at which homologs are located

139 then yields the designation "Taxonomically Restricted Gene" (TRG) for the cluster of orthologs

140 (homologs) captured by the classifier. If no orthologous sequences are found, and the ORF remains a

141 species-unique sequence, it will be classified as an "Orphan Gene." If the ORF is unique at the

142 subspecies level, the gene is classified as a "Strict Orphan" by ORFanID. Using this algorithm, 
ORFanID: Web-Based Search Engine

143 ORFanID identifies and displays orphan genes or TRGs. Results can be viewed and analyzed

144 graphically. Further details on the ORFanID algorithm are provided in the Supplementary Information

145 section of this publication.

\section{$146 \quad 2.2$ Operation}

147 ORFanID accepts either the protein or amino acid sequences of a single or multiple gene

148 sequences in the FASTA format. Users can easily retrieve numerous genes or protein sequences by

149 providing multiple accessions to the sequence search engine. Currently, ORFanID supports both NCBI

150 as well as Uniprot accessions. Optionally, the user can upload a FASTA file or directly copy the

151 sequence into the ORFanID engine according to specifications provided on the ORFanID website.

152 Secondly, users can select the species from the dropdown menu, containing species scientific names,

153 the NCBI taxonomy ID, and an image of the species for convenient visual recognition. Finally, by

154 using the advanced parameters, the accuracy of the results can be fine-tuned based on the E-value and

155 a maximum number of target sequences for each BLAST search. Default values for the advanced

156 parameters are as follows: max target sequences, 500; identity 60\% (minimum percent similarity of

157 alignment); expected value, 10-3; since most cited papers use this value.

158 As Figure 4 illustrates, four distinct examples are provided to new users for demonstrative

159 purposes. Once the user submits their sequence and the species, along with the advanced parameters,

160 ORFanID will execute the steps discussed in the Algorithm section above and display results in Figure

161 5A. Figure 5A, the top-left table, summarizes the metadata of the analysis. The graph on the top right

162 depicts the taxonomy of the TRG or orphan gene for convenient visualization. The table at the bottom

163 shows the categorization levels of the genes discovered, along with the actual taxonomy level for each 164 gene.

165 The homology results can be viewed graphically by selecting any of the graph icons at the 166 bottom of the results page (Figure 5B). The number of BLAST matches for each taxonomic rank is 


\section{ORFanID: Web-Based Search Engine}

167 visualized in this interactive chart. Each node in the tree shows the related orthologs found at each

168 taxonomic rank. The graphical display is expandable and collapsible by taxonomy level. All the tables

169 on the results page are sortable by column, and the results can be easily filtered by fuzzy search

170 functionality. Besides its web service, ORFanID can be downloaded and installed on a local server.

171 The Source-code and installation instructions are freely available online.

172 At the user experience level, ORFanID is equipped with a dedicated web page that outlines the

173 installation and operating instructions. The site also includes video tutorials that will help users

174 understand its web interface. As noted previously, the sequence submissions page also provides four

175 example sets of input gene data for demonstrational purposes. They are available both as FASTA file

176 sequences and as NCBI accession numbers. These four examples analyze sequences of the following

177 species: Escherichia coli (562), Drosophila melanogaster (7227), Homo sapiens (9606), and

178 Arabidopsis thaliana (3702). The operation of the ORFanID application is architected for ease of use,

179 rich graphics, and reasonable speed for providing a research instrument to identify orphan genes at all

180 taxonomic levels.

\section{3. Results and Evaluations}

We tested the functionality of ORFanID by analyzing the protein and DNA sequences (or NCBI accessions) of various prokaryotic and eukaryotic organisms, such as E. coli, C. elegans, S. cerevisiae, D. melanogaster, and H. sapiens (Supplementary Table 1). We adjusted the parameters (e-value $<=10^{3}$ ) to filter low-quality results (transposable elements, low complexity protein regions, etc.) from our analyses. Our results show that the ORFanID effectively assigns the protein accessions to their respective taxonomic levels (Supplementary Table 1). For instance, paired box-containing pax-6 proteins are tissue-specific transcriptional factors highly conserved across most animal phyla. As

189 expected, testing the Drosophila melanogaster Pax-6 proteins, Eyeless (NP_726607.1) and Twin of

190 eyeless (NP_001259080.1) using ORFanID, showed that these proteins are restricted to phylum and 191 class levels. 
ORFanID: Web-Based Search Engine

\section{SPANX (NP_073152.2). N-cym protein regulates the stability of the proto-oncogene MYCN in}

194 neuroblastoma cells. In contrast, the SPANX family proteins are expressed in human spermatozoa and

195 are extensively studied in Down syndrome patients (Salemi et al. 2009). A previous study predicted

196 that these two proteins are restricted to the order Primates (Kouprina et al. 2007). Surprisingly,

197 ORFanID showed that only N-cym is order restricted, while SPANX was classified as a family

198 restricted protein. To understand this disparity, we searched OrthoDB (Kriventseva et al. 2019) and the

199 non-redundant database of NCBI using H. sapiens SPANX protein (NP_073152.2) as the query.

200 Interestingly, both databases show that SPANX proteins are restricted to the Hominidae family. These

201 results support that the ORFanID algorithm is accurate in classifying proteins to their respective

202 taxonomic group based upon the functionality of BLAST and the choice of parameter settings.

203 Next, to accurately identify species-specific orphan genes, we tested genes from various species

204 previously shown to be orphans by using ORFanID. The $A$. thaliana $Q Q S$ gene is one of the first plant

205 genes shown to be a species-level orphan ( $\mathrm{Li}$ et al. 2009). It is involved in the regulation of starch

206 biosynthesis in leaves ( $\mathrm{Li}$ et al. 2009), increasing of seed protein, and enhancing the resistance to

207 pathogens (Qi et al. 2018). Our results show that ORFanID accurately classifies QQS protein

208 (NP_189695.1) as an A. thaliana specific protein. Similarly, we tested the species-specific orphan

209 genes of some model organisms, such as D. melanogaster, C. elegans, and S. cerevisiae. ORFanID

210 precisely identified these published genes as species-specific orphans. The D. melanogaster group-

211 specific orphan genes, jeanbaptiste, and karr were predominantly expressed in the male germline and

212 are functionally very important because RNAi-mediated knock-down of these genes lead to male-

213 specific developmental defects and partial-lethality (Reinhardt and Jones 2013). As we expected,

214 ORFanID grouped these genes as strict species-level orphans. In S. cerevisiae, the de novo genes bsc4

215 and fyv5, which regulate DNA repair and vegetative growth, respectively, were accurately predicted

216 as species-specific by ORFanID. Finally, we analyzed the C. elegans-specific gene ify-1 required for 
ORFanID: Web-Based Search Engine

217 proper chromosome segregation during cell division. As expected, ORFanID rightly categorized ify-1

218 as a species-specific protein. This result was further confirmed by the database "wormbase.org” (Harris

219 et al. 2020), which did not show any orthologous of the ify-1 gene. Taken together, these results suggest

220 that ORFanID works accurately and reliably in classifying and identifying species-specific orphan

221 genes. Refer to Supplemental Information for a complete list of taxonomically restricted or species-

222 specific orphans we tested using ORFanID.

Nonetheless, as the NCBI and other such databases grow, what is currently classified as an

orphan may be re-classified at another level in their taxonomic group. Furthermore, the database of

orphans and TRGs is a dynamic list. As such, Orphans may have a provisional status and thus change

as sampling grows. However, we noticed over the years that the percentage of orphans in every species

tends to stabilize with the growing number of genomes synthesized. Hence, ORFanID regularly updates

the databases and files obtained from NCBI.

We compared ORFanFinder to ORFanID, resulting from genes from five different organisms

(Caenorhabditis elegans, Escherichia coli, Homo sapiens, Oryza sativa, and Zea mays). Results show

that ORFanID is more sensitive in classifying orphan and strict-orphan genes (ORFanFinder is no

longer updated, and the last version of its database is from 2018). Refer to Supplemental Table 2 for more details.

We also compared ORFanID with abSENSE (Weisman et al. 2020) and found that ORFanID

classifies only $68 \%$ of the Fungal genes as orphans and $90 \%$ of the Insect. This attests that ORFanID

which a percentage of orphans may prove not to be orphans when more organisms are sequenced and

\section{Discussion}

Here we present the implementation of a web-based, easy-to-use intuitive tool helpful in

241 identifying and discovering orphan genes across any taxonomic lineage by geneticists/biologists, 
ORFanID: Web-Based Search Engine

242 bioinformaticians, and computational biologists. The search engine is designed to require minimal

243 computational skills to operate when compared to other bioinformatics programs. This web-based

244 interactive tool is based on the BLAST database and uses the genomic sequences for various species

245 made available through NCBI (Fischer and Eisenberg 1999) and (Yin and Fischer 2006). While

246 previous stand-alone programs such as ORFanFinder have existed in the past, their scope was limited

247 mainly to protein databases. To our knowledge, this is the first program that allows users to identify

248 orphan genes and TRGs using both nucleotide and protein sequences; and from ORFanID, any taxon

249 of interest found in the NCBI databases can be classified.

250 Taken together with its interactive user interface, ORFanID allows users to seamlessly use the

251 BLAST database to investigate and categorically identify orphan genes. These findings can lead to the

252 creation of databases of these clandestine genes pre-identified at the various taxonomic levels as

253 ascertained by ORFanID, leading to a much deeper understanding of the purpose of orphan genes, their

254 function in genomes, and their potential impact on life. 


\section{Author Contributions}

ORFanID: Web-Based Search Engine

258 RG conceptualized and led the project along with PN. SH and VG architected and developed the software. KR tested the program and helped with writing. TG and ET helped with writing and editing. VG managed the software team. The authors declare that there is no conflict of interest.

\section{Funding}

263 This work was supported in part by the Peter \& Carla Roth Family.

\section{Acknowledgments}

266 We acknowledge the programming work of intern Savidu Dias, video creations by intern Megan Rupp,

267 and research fellow Jesse Gentile, including technical support of engineering interns Kenneth Chen

268 and Daniel Dirksen. We thank Marisa Arthur for her expert editorial assistance. The support of the

269 Peter \& Carla Roth Family for the interns, and the expert technical work of Chesalon USA, Inc. is

270 greatly appreciated.

271

\section{Supplementary Information}

273 Supplementary data are available in the online repository of the journal. The Supplementary Material

274 for this article can be found online at: (please insert the link when ready)

\section{Data Availability and Implementation Statement}

277 The ORFanID Web Application and Database are freely available to non-commercial users at 278 http://www.orfangenes.com/. The Development Stack of ORFanID comprises a VueJs front-end, a

279 back-end in the Spring Boot framework, and a database in PostgreSQL. The software modules of 280 ORFanID are containerized using Docker and communicate using RESTful APIs. ORFanID supports 281 all major browsers. 
bioRxiv preprint doi: https://doi.org/10.1101/2022.02.01.478498; this version posted February 4, 2022. The copyright holder for this preprint (which was not certified by peer review) is the author/funder. All rights reserved. No reuse allowed without permission.

ORFanID: Web-Based Search Engine

282 The Source Code for ORFanID is freely available at https://github.com/orfanid 
References

286 Altenhoff, A., Glover, N., Train, C.M., Kaleb, K., Vesztrocy, A.W., Dylus, D., Farias, T., Zile, K.,

287 Stevenson, C., Long, J., Redestig, H., Gonnet, G.H., Dessimoz, C. 2018. The OMA orthology

288 database in 2018: retrieving evolutionary relationships among all domains of life through richer web

289 and programmatic interfaces. Nucleic Acids Research 46 (D1): D477-D485.

290 doi:10.1093/nar/gkx1019

291 Buchfink, B., Xie, C., Huson, D. 2015. Fast and Sensitive Protein Alignment using DIAMOND. Nature

292 Methods 12: 59-60. doi:10.1038/nmeth.3176

293 Domazet-Lošo, T., Brajković, J., Tautz, D. 2007. A phylostratigraphy approach to uncover the genomic

294 history of major adaptations in metazoan lineages. Trends in Genetics 23

295

(11): 533-539. doi:10.1016/j.tig.2007.08.014

Ekstrom, A., and Yin, Y. 2016. ORFanFinder: automated identification of taxonomically restricted orphan genes. Bioinformatics 32 (13): 2053-2055. doi:10.1093/bioinformatics/btw122

Emms, D.M., and Kelly, S. 2019. OrthoFinder: phylogenetic orthology inference for comparative genomics. Genome Biology 20 (238 ). doi:10.1186/s13059-019-1832-y

300 Fischer, D., and Eisenberg, D. 1999. Finding families for genomic ORFans. Bioinformatics 15 (9):

302 Gao, Q., Xiu, J., Xia, E., Wu, X., Gu, L., Yan, H., Xia, Y., Li, S. 2020. Identification of Orphan Genes

303 in Unbalanced Datasets Based on Ensemble Learning. Frontiers in Genetics (Systems Biology Archive)

304 11: 820. doi:10.3389/fgene. 2020.00820

305 Harris, T., Arnaboldi, T., Cain, S., Chan, J., Chen, W., Cho, J., Davis, P., Gao, S., Grove, C.A., Kishore,

306 R., Lee, R.Y.N., Muller, H.M., Nakamura, C., Nuin, P., Paulini, M., Raciti, D., Rodgers, F.H., Russell, 
ORFanID: Web-Based Search Engine

307 M., Schindelman, G., Auken, K.V., Wang, Q., Williams, G., Wright, A.J., Yook, K., Howe, K.L.,

308 Schedl, T., Stein, L., Sternberg, P.W. 2020. WormBase: a modern Model Organism Information

309 Resource. Nucleic Acids Research 48 (D1): D762-D767. doi:10.1093/nar/gkz920

310 Ibrahim, A., Colson, P., Merhej, V., Zgheib, R., Maatouk, M., Naud, S., Bittar, F., Raoult, D. 2021.

311 Rhizomal Reclassification of Living Organisms. International Journal of Molecular Sciences 22 (11):

312 5643. doi:10.3390/ijms22115643

313 Johnson, B.R., and Tsutsui, N.D. 2011. Taxonomically restricted genes are associated with the

314 evolution of sociality in the honey bee. BMC Genomics 12 (164). doi:10.1186/1471-2164-12-164

315 Johnson, B. 2018. Taxonomically Restricted Genes Are Fundamental to Biology and

316 Evolution. Frontiers in Genetics (Evolutionary and Population Genetics) 9: 407.

317 doi:10.3389/fgene.2018.00407

318 Kearse, M., Moir, R., Wilson, A., Stones-Havas, S., Cheung, M., Sturrock, S., Buxton, S., Cooper,

319 A., Markowitz, S., Duran, C., Thierer, T., Ashton, B, Meintjes, P., Drummond, A. 2012. Geneious

320 Basic: An integrated and extendable desktop software platform for the organization and analysis of

321 sequence data. Bioinformatics 28 (12): 1647- 1649. doi:10.1093/bioinformatics/bts199

322 Khalturin, K., Georg, H., Sebastian, F., René, A., Bosch, T.C.G. 2009. More than just orphans: are

323 taxonomically-restricted genes important in evolution? Trends in Genetics 25 (9): 404-413.

324 doi:10.1016/j.tig.2009.07.006

325 Kouprina, N., Noskov, V., Pavlicek, A., Collins, K., Bortz, P., Ottolenghi, C., Loukinov, D.,

326 Goldsmith, P., Risinger, J.I., Kim, J.H., Westbrook, V.A., Solomon, G., Sounders, H., Herr, J.C., Jurka,

327 J., Lobanenkov, V., Schlessinger, D., Larionov, V. 2007. Evolutionary Diversification of SPANX-N

328 Sperm Protein Gene Structure and Expression. PLOS ONE. doi:10.1371/journal.pone.0000359 


\section{ORFanID: Web-Based Search Engine}

329 Kriventseva, E.V., Kuznetsov, D., Tegenfeldt, F., Manni, M., Dias, R., Simão, F.A., Zdobnov, E.M.

330 2019. OrthoDB v10: sampling the diversity of animal, plant, fungal, protist, bacterial and viral

331 genomes for evolutionary and functional annotations of orthologs. Nucleic Acids Research 8 (47):

332 D807D811. doi:10.1093/nar/gky1053

333 Li, L., Foster, C.M., Gan, Q., Nettleton, D., James, M.G., Myers, A.M., Wurtele, E.S. 2009.

334 Identification of the novel protein QQS as a component of the starch metabolic network in

335 Arabidopsis leaves. Plant Journal 58 (3): 485-498. doi:10.1111/j.1365-313X.2009.03793.x

336 Li, L., Stoeckert, C.J. Jr, Roos, D.S. 2003. OrthoMCL: Identification of Ortholog Groups for

337 Eukaryotic Genomes. Genome Research (Cold Spring Harbor Laboratory Press) (13):

338 2178-2189. doi:10.1101/gr.1224503

339 Lupas, A.N., Ponting, C.P., Russell, R.B. 2001. On the evolution of protein folds: are similar motifs

340 in different protein folds the result of convergence, insertion, or relics of an ancient peptide world?

341 Journal of Structural Biology 134 (2-3): 191-203. doi:10.1006/jsbi.2001.4393

342 Mirdita, M., Steinegger, M., Söding, J. 2019. MMseqs2 desktop and local web server app for fast,

343 interactive sequence searches. Bioinformatics 35 (16): 2856-2858.

344 doi:10.1093/bioinformatics/bty1057

345 Prabh, N, Rödelsperger, C. 2016. Are orphan genes protein-coding, prediction artifacts, or non-coding 346 RNAs? Bioinformatics 17 (226). doi:10.1186/s12859-016-1102-x

347 Priyam, A., Woodcroft, B.J., Rai, V., Moghul, I., Munagala, A., Ter, F.,

348 Chowdhary, H., Pieniak, I., Maynard, L.J., Gibbins, M.A., Moon, H.K., Davis-Richardson, A., Uludag,

349 M., Watson-Haigh, N.S., Challis, R., Nakamura, H., Favreau, E., Gómez, E.A., Pluskal, T., Leonard, 350 G., Rumpf, W., Wurm, Y. 2019. Sequenceserver: A Modern Graphical User Interface for Custom 
351 BLAST Databases. Molecular Biology and Evolution 36 (12): 2922-2924.

352 doi:10.1093/molbev/msz185

353 Qi, M., Wenguang, Z., Zhao, X., Hohenstein, J., Kandel, Y., O'Conner, S., Wang, Y., Du, C.,

354 Nettleton, D., MacIntosh, G.C., Tylka, G.L., Wurtele, E.S., Whitham, S.A., Li, L. 2018. QQS orphan

355 gene and its interactor NF-YC4 reduce susceptibility to pathogens and pests. Plant Biotechnology

356 Journal 17 (1): 252-263. doi:10.1111/pbi.12961

357 Reinhardt, J.A., Jones, C.D. 2013. Two rapidly evolving genes contribute to male fitness in

358 Drosophila. Journal of Molecular Evolution 77 (5-6): 246-59. doi:10.1007/s00239-013-9594-8

359 Salemi, M., Romano, C., Barone, C. et al. 2009. SPANX-B and SPANX-C (Xq27 region) gene dosage

360 analysis in Down's syndrome subjects with undescended testes. Journal of Genetics 88: 93-

361 97. doi:10.1007/s12041-009-0013-2

362 Tautz, D., Domazet-Lošo, T. 2011. The evolutionary origin of orphan genes. Nature Reviews Genetics

363 12 (10): 692-702. doi:10.1038/nrg3053

364 Tautz, D. 2011. Not just another genome. BMC Biology 9 (8). doi:10.1186/1741-7007-9-8

365 Weisman, C.M., Murray, A.W., Eddy, S.R. 2020. Many, but not all, lineagespecific genes can be

366 explained by homology detection failure. PLoS Biology 18 (11). doi:10.1371/journal.pbio.3000862

367 Wissler, L., Gadau, J., Simola, D.F., Helmkampf, M., Bornberg-Bauer, E. 2013. Mechanisms and

368 Dynamics of Orphan Gene Emergence in Insect Genomes. Genome biology and evolution 5 (2): 439-

369 455. doi:10.1093/gbe/evt009

370 Yin, Y., Fischer, D. 2006. On the origin of microbial ORFans: quantifying the strength of the evidence

371 for viral lateral transfer. BMC Evolutionary Biology 6 (63). doi:10.1186/1471-2148-6-63 
bioRxiv preprint doi: https://doi.org/10.1101/2022.02.01.478498; this version posted February 4, 2022. The copyright holder for this preprint (which was not certified by peer review) is the author/funder. All rights reserved. No reuse allowed without permission.

ORFanID: Web-Based Search Engine

372 Yu, G., Stoltzfus, A. 2012. Population diversity of ORFan genes in Escherichia coli. Genome

373 Biology and Evolution 4 (11): 1176-87. doi:10.1093/gbe/evs081 


\section{ORFanID: Web-Based Search Engine}

\section{Figures}

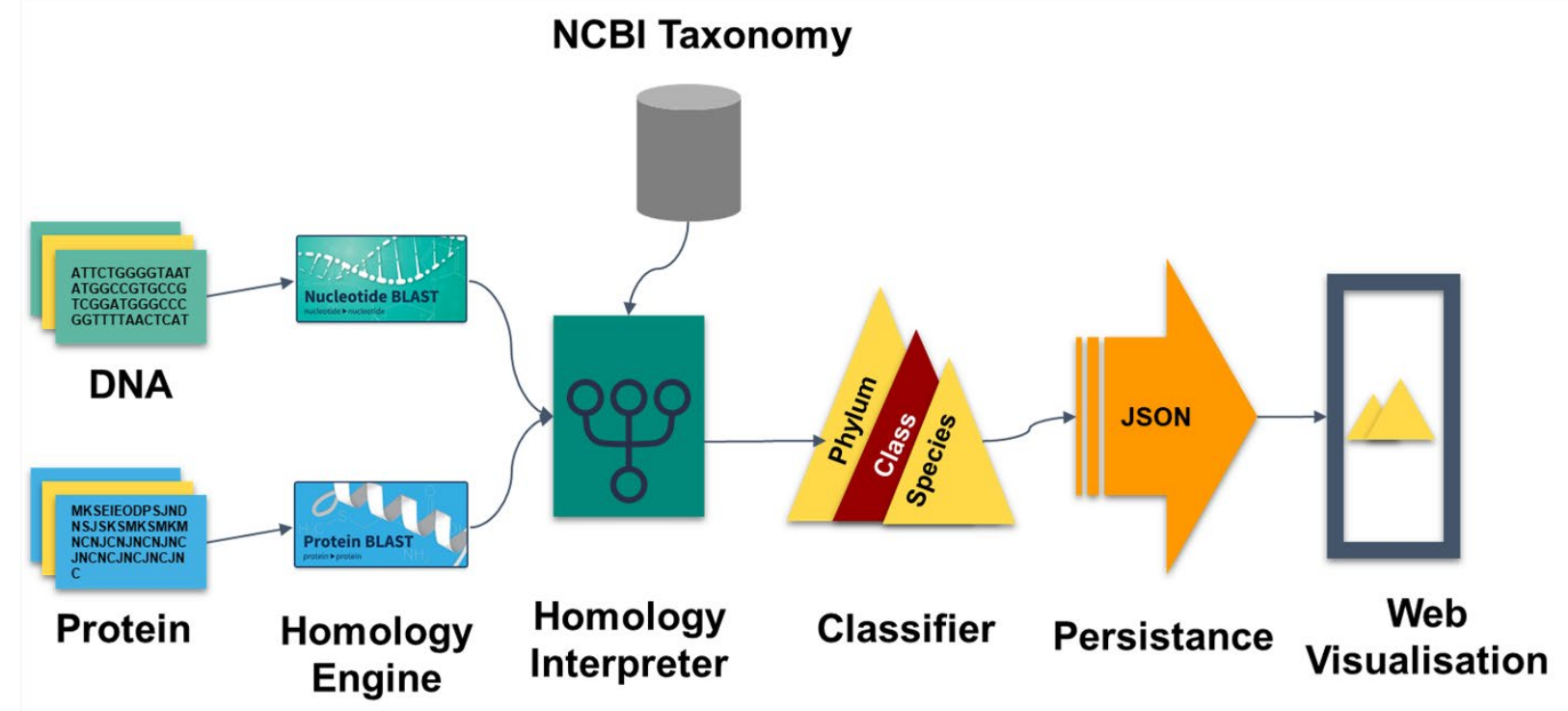

378 Figure 1: Core engine of ORFanID

379 ORFanID is a web-based application developed on Java Spring Framework. The Web UI was

380 developed in Thymeleaf and MaterializedCSS, which follows Google Material design concepts. 


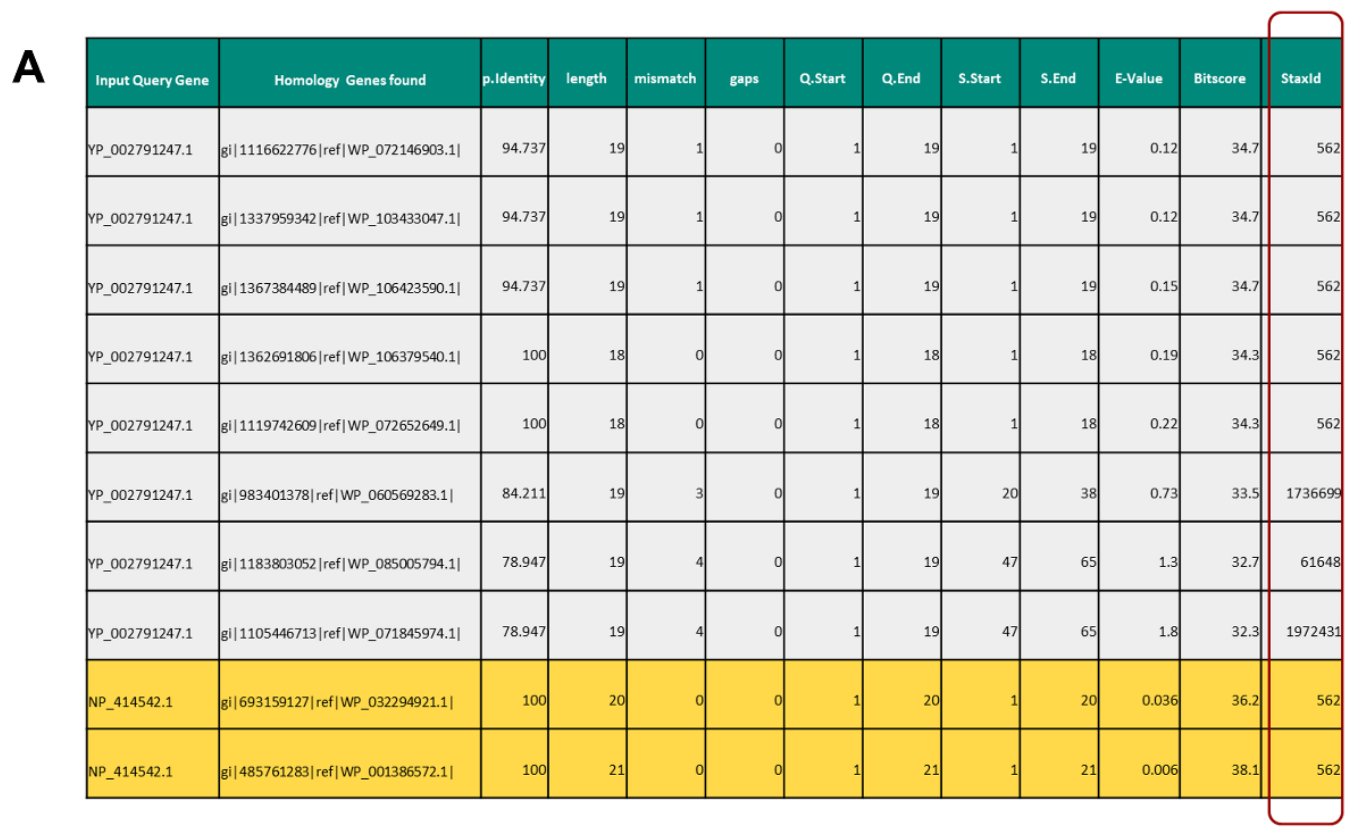

B

\begin{tabular}{|c|c|c|c|c|c|c|c|c|c|}
\hline $\begin{array}{c}\text { Taxonomy } \\
\text { Id }\end{array}$ & $\begin{array}{c}\text { Scientific } \\
\text { name }\end{array}$ & $\begin{array}{l}\text { Species } \\
\text { name }\end{array}$ & $\begin{array}{l}\text { genus } \\
\text { name }\end{array}$ & $\begin{array}{l}\text { family } \\
\text { name }\end{array}$ & $\begin{array}{l}\text { order } \\
\text { name }\end{array}$ & $\begin{array}{c}\text { class } \\
\text { name }\end{array}$ & $\begin{array}{l}\text { Phylum } \\
\text { name }\end{array}$ & kingdom name & $\begin{array}{c}\text { Superkingdom } \\
\text { name }\end{array}$ \\
\hline 562 & Escherichia coli & N/A & Escherichia & Enterobacteriaceae & Enterobacterales & Gammaproteobacteria & Proteobacteria & $\mathrm{N} / \mathrm{A}$ & Bacteria \\
\hline 562 & Escherichia coli & N/A & Escherichia & Enterobacteriaceae & Enterobacterales & Gammaproteobacteria & Proteobacteria & $\mathrm{N} / \mathrm{A}$ & Bacteria \\
\hline 562 & Escherichia coli & N/A & Escherichia & Enterobacteriaceae & Enterobacterales & Gammaproteobacteria & Proteobacteria & N/A & Bacteria \\
\hline 562 & Escherichia coli & N/A & Escherichia & Enterobacteriaceae & Enterobacterales & Gammaproteobacteria & Proteobacteria & $\mathrm{N} / \mathrm{A}$ & Bacteria \\
\hline 562 & Escherichia coli & N/A & Escherichia & Enterobacteriaceae & Enterobacterales & Gammaproteobacteria & Proteobacteria & $\mathrm{N} / \mathrm{A}$ & Bacteria \\
\hline 1736699 & Citrobacter sp. 50677481 & N/A & Citrobacter & Enterobacteriaceae & Enterobacterales & Gammaproteobacteria & Proteobacteria & N/A & Bacteria \\
\hline 61648 & Kluyvera intermedia & N/A & Kluyvera & Enterobacteriaceae & Enterobacterales & Gammaproteobacteria & Proteobacteria & $\mathrm{N} / \mathrm{A}$ & Bacteria \\
\hline 1972431 & Phytobacter ursingii & N/A & Phytobacter & $N / A$ & Enterobacterales & Gammaproteobacteria & Proteobacteria & $N / A$ & Bacteria \\
\hline 562 & Escherichia coli & N/A & Escherichia & Enterobacteriaceae & Enterobacterales & Gammaproteobacteria & Proteobacteria & $\mathrm{N} / \mathrm{A}$ & Bacteria \\
\hline 562 & Escherichia coli & N/A & Escherichia & Enterobacteriaceae & Enterobacterales & Gammaproteobacteria & Proteobacteria & $N / A$ & Bacteria \\
\hline
\end{tabular}

383 Figure 2: (A) Finding homologous genes by sequence similarity. (B) Finding taxonomy levels for each homology. 
bioRxiv preprint doi: https://doi.org/10.1101/2022.02.01.478498; this version posted February 4, 2022. The copyright holder for this preprint (which was not certified by peer review) is the author/funder. All rights reserved. No reuse allowed without permission.

ORFanID: Web-Based Search Engine

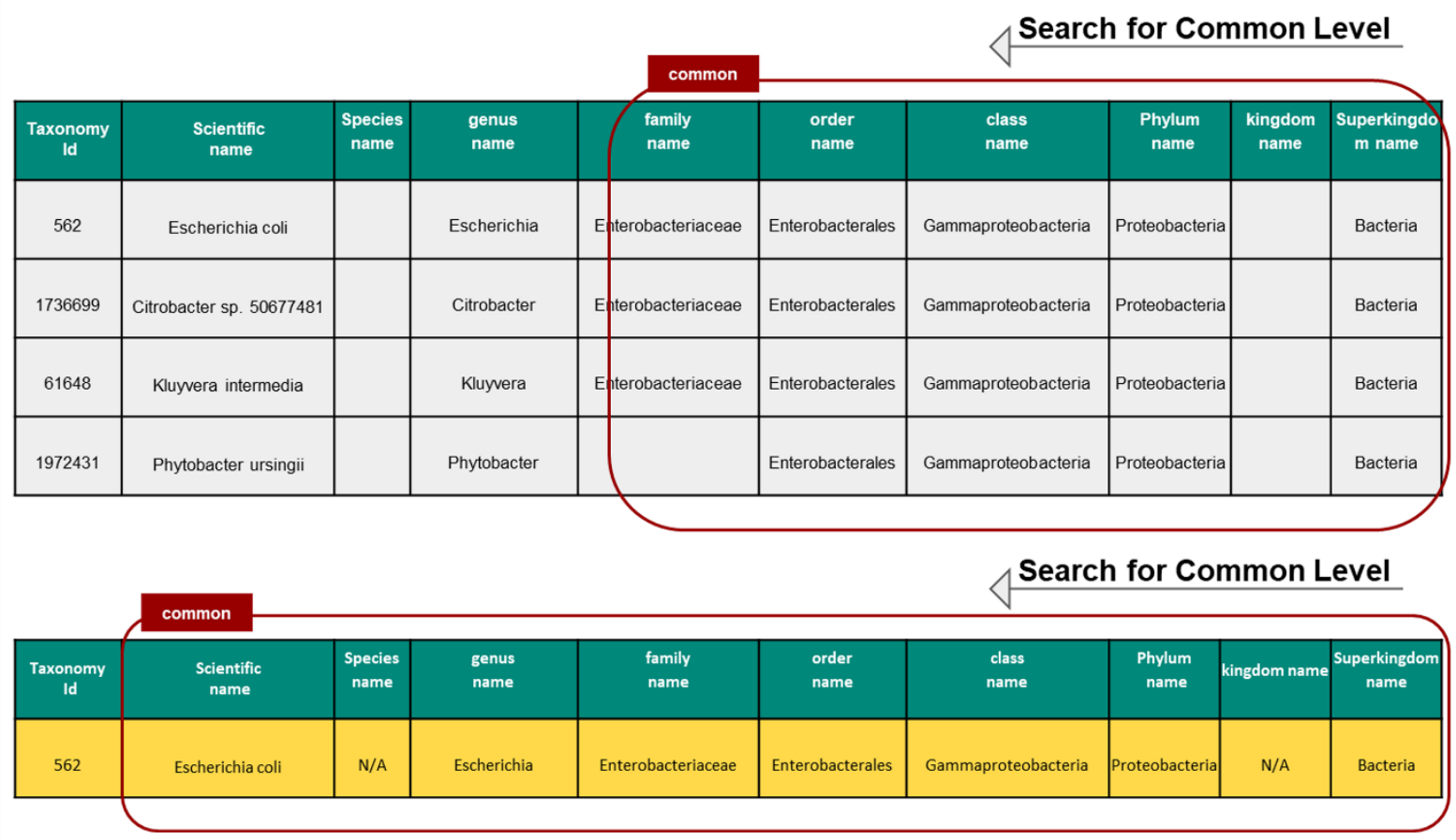

388 Figure 3: Gene Classification. The search starts from the superkingdom to species level until it

389 finds homologous sequences.

390

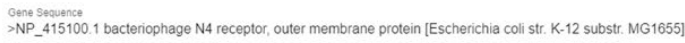

MKENNLNRVIGWSGLLTSLLSTSALADNGTSAEELGLSDYRHFVYYPRLDKALKAOKNNDEATAREF

EYYHQQVPDNPITTYYLAEAYRHFGHDDRARLLLEDQLKRHPGDARLERSLAAIPVEVKSVTTVEELLAQ
QKACDAAPLRCRSEVGQNALRLAQLPVARAQLNDATFAASPEGKTLRTDLLQRAIYLKOWSOADTLYNE

ARQQNTLSAAERRQWFDVLLAGQLDDRLLLLSQGIFTDPQSYITYATALAYRGEKARLOHYLENKPLF

Orgensm
Escherichia coll(1562)

Search By: sequence $\Psi$ accessions

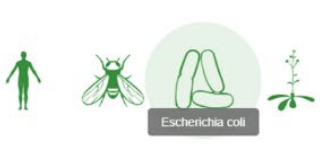

Figure 4: ORFanID Input Page. 
bioRxiv preprint doi: https://doi.org/10.1101/2022.02.01.478498; this version posted February 4, 2022. The copyright holder for this preprint (which was not certified by peer review) is the author/funder. All rights reserved. No reuse allowed without permission.

ORFanID: Web-Based Search Engine
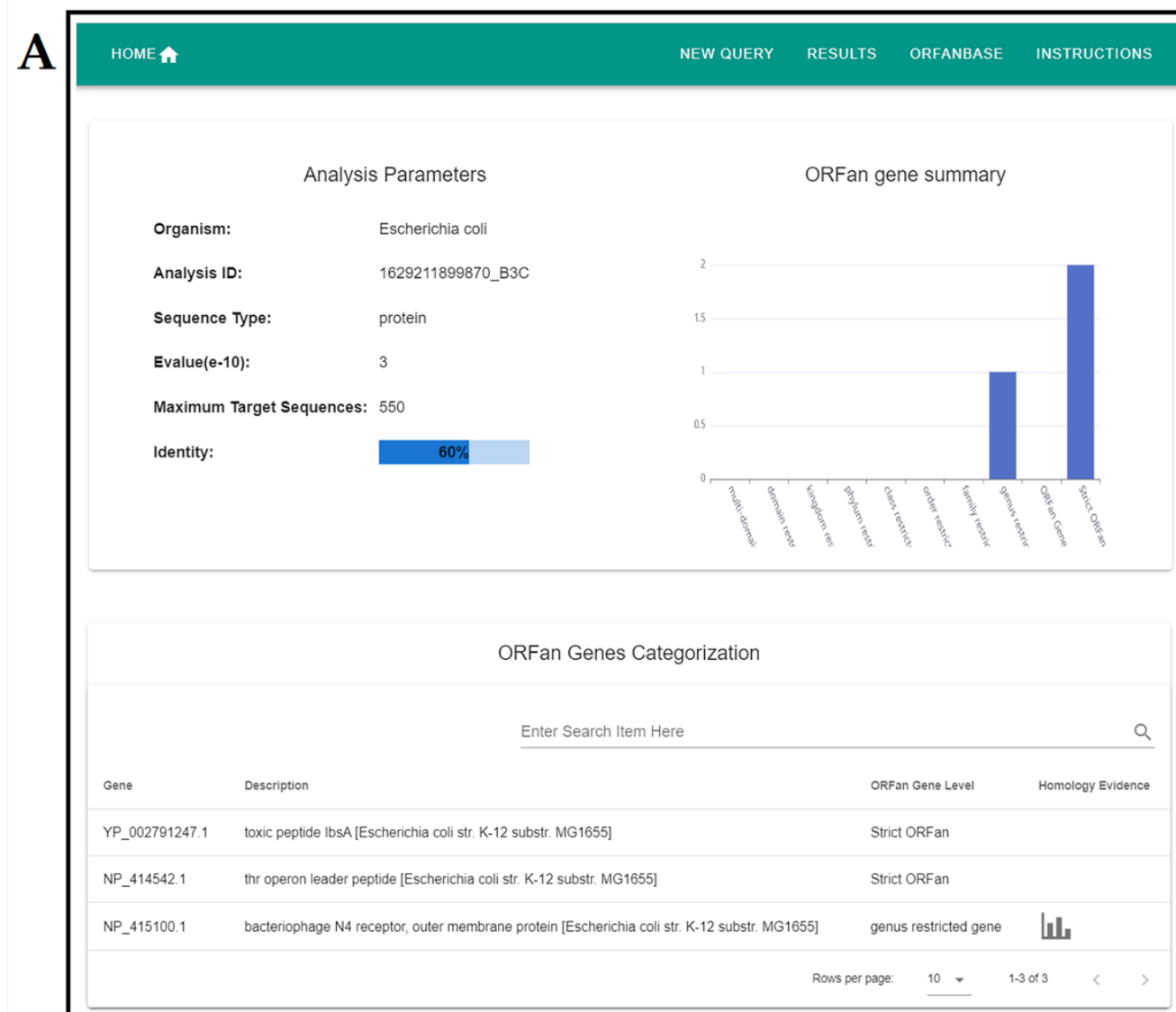

$\underline{ \pm}$ ELAST RESULTS

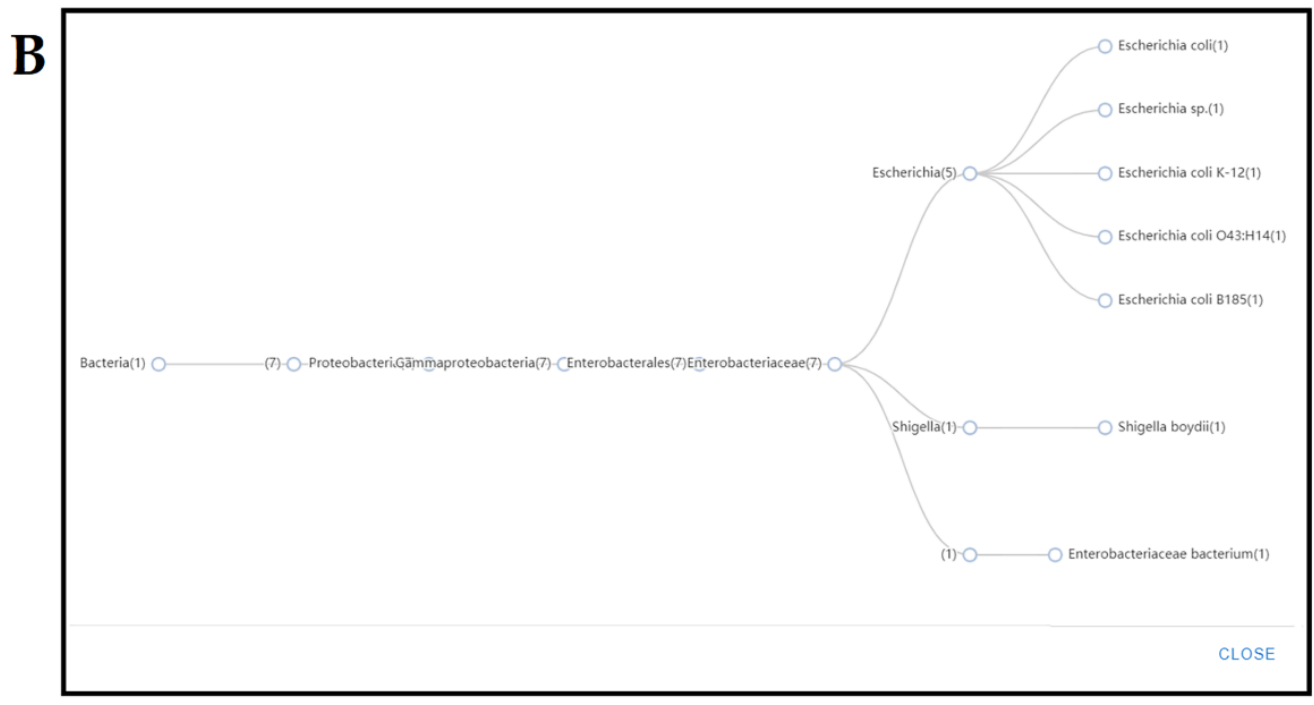

395 Figure 5: (A) ORFanID Results Page. (B) ORFanID BLAST Results Graphical Display. 